\title{
Multivariate Image Analysis, Histogram Matching and Statistical Process Control Chart Assisted Nucleation Detection of Pharmaceutical Crystallization processes
}

\author{
L. L. SimON ${ }^{1}$, K. AbBou OUCheriF ${ }^{2}$, Z. K. NAGY ${ }^{3}$, K. HUNGerbuhler ${ }^{1}$ \\ ${ }^{1}$ ETH Zurich, Institute of Chemical and Bioengineering, Zurich, Switzerland \\ ${ }^{2}$ New-Mexico Institute of Technology, Department of Chemical Engineering, Socorro, USA \\ ${ }^{3}$ Loughborough University, Chemical Engineering Department, Loughborough, United Kingdom \\ E-mail: Levente.simon@chem.ethz.ch (L. L. Simon)
}

Sci Pharm. 2010; 78: 665

doi:10.3797/scipharm.cespt.8.PMS38

\begin{abstract}
The aim of this work is to investigate image processing and process monitoring technologies which can provide a systematic, robust and sensitive solution for nucleation detection using bulk video imaging (BVI). The external BVI method is based on a video camera and a capture hardware, which captures 25 frames of the crystallization bulk per second [1]. Another set of experimental data is generated using in-situ endoscopy and a small scale crystallization calorimeter [2]. In this work the images are converted into time series and these are monitored using Shewhart and exponentially moving average (EWMA) statistical process control (SPC) charts; nucleation is detected when the signals exceed the control limits. A second approach to nucleation detection is histogram matching. Histograms of the images are generated and similarity measures are applied to detect significant changes indicating the beginning of nucleation; the current study evaluates the use of Kolmogorov-Smirnof and $\mathrm{Chi}^{2}$ statistics to determine the statistically significant change between the observed and the reference histograms for a 95\% confidence interval.

The third approach relies on the advantages offered by the multivariate image analysis [3]. According to this, multiway principal component analysis (PCA) is performed on the color images to obtain the first score, which is also an image. Furthermore, the PCA model residual monitoring performance was investigated. The image analysis techniques are applied for the crystallization of caffeine as a model for active pharmaceutical ingredients, both externally and in-situ.
\end{abstract}

[1] Simon LL, Nagy ZK, Hungerbuhler K. Comparison of external bulk video imaging with focused beam reflectance and ultra violet-visible spectroscopy for crystallization nucleation detection and metastable zone identification. Chem Eng Sci. 2009; 64: 3344-3351. doi:10.1016/j.ces.2009.04.016

[2] Simon LL, Nagy ZK, Hungerbuhler K. Endoscopy-Based in Situ Bulk Video Imaging of Batch Crystallization Processes. Org Process Res Dev. 2009; 13: 1254-1261. doi:10.1021/op900019b

[3] Simon LL, Abbou Oucherif K, Nagy ZK, Hungerbuhler K. Bulk video imaging based multivariate image analysis, process control chart and acoustic signal assisted nucleation detection. Chem Eng Sci. 2010;65:4983-4995. doi:10.1016/j.ces.2010.05.045 